\title{
The LAMOST Complete Spectroscopic Survey of Pointing Area at Southern Galactic Cap
}

\author{
Hong $\mathbf{W u}^{1}$, Ming Yang ${ }^{1,2}$, Man I Lam ${ }^{1,3}$, Fan Yang ${ }^{1}$, Chao-Jian $\mathbf{W u}{ }^{1}$, \\ Tian-Wen $\mathrm{Cao}^{1}$ and LAMOST Collaboration \\ ${ }^{1}$ Key Laboratory of Optical Astronomy, National Astronomical Observatories, Chinese \\ Academy of Sciences, 20A Datun Road, Chaoyang District, Beijing, 100012, China \\ email: hwu@bao.ac.cn \\ ${ }^{2}$ IAASARS, National Observatory of Athens, Vas. Pavlou \& I. Metaxa, Penteli 15236, Greece \\ email: myang@noa.gr \\ ${ }^{3}$ Shanghai Astronomical Observatory, Chinese Academy of Sciences, Shanghai 200030, China
}

\begin{abstract}
The LAMOST Complete Spectroscopic Survey of Pointing Area (LCSSPA) at Southern Galactic Cap (SGC), is one of the LAMOST Key Project, designed to complete the spectroscopic observations of all Galactic and extra-galactic sources in two selected fields of 20 degrees ${ }^{2}$ at SGC, with the limiting magnitude of $r=18.1 \mathrm{mag}$. The main purposes of the project are focused on the completeness of the LAMOST ExtraGAlactic Surveys (LEGAS), testing the selection methods of galaxies and stars, and obtaining the basic performance parameters of the LAMOST telescope. Meanwhile the scientific studies include galaxies, clusters of galaxies, variable sources (quasars and variable stars), infrared excess stars and luminous infrared galaxies. The project has considerable scientific value since it is the most complete spectral data in LEGAS up to now. The project completed its observation in the early of 2014, and obtained at least 5000 spectra of galaxies and 25000 spectra of stars.
\end{abstract}

Keywords. catalog, surveys, galaxies: distances and redshifts, galaxies: clusters: general

\section{Overviews of the LCSSPA Project}

As an important part of LAMOST scientific survey, LAMOST ExtraGAlactic Surveys (LEGAS) aims to take hundreds of thousands of spectra for extra-galactic objects over 8000 degrees $^{2}$ of the Northern Galactic Cap (NGC) and 3500 degrees $^{2}$ of the Southern Galactic Cap (SGC) in five years. As a part of the LEGAS, the LCSSPA project focuses on the completeness of the galaxy survey. It also provides an opportunity to carry out different scientific studies from stars to galaxies, such as galaxies, clusters of galaxies, variable sources (quasars and variable stars), infrared excess stars and luminous infrared galaxies, etc.

Two selected fields of 20 degree $^{2}$ are located at $R . A .=37.88^{\circ}, D e c .=3.44^{\circ}$ (hereafter Field A) and R.A. $=21.53^{\circ}$, Dec. $=-2.20^{\circ}$ (hereafter Field B) at SGC, and represent relatively lower and higher density regions of galaxies, based on the distribution of galaxy clusters of Abell rich galaxy clusters catalog (Abell et al. 1989). The fields are also located in the region of the South Galactic Cap u-band Sky Survey (SCUSS, Zou et al. 2015 ).

The targets are constituted by all the stars and galaxies from SDSS DR9 photometric data with r-band magnitude limit of 18.1, quasar candidates selected by the opticalinfrared color-color criteria (Wu \& Jia 2010, Wu et al. 2012), and u-band variable candidates selected by SDSS and SCUSS u-band catalogues. 


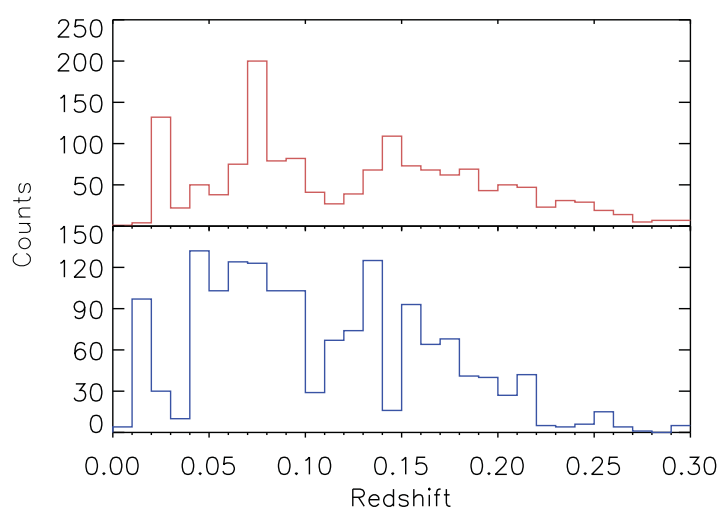

Figure 1. The redshift distributions of Field A (top) and B (bottom).

\section{Observations and Data Reduction}

The observations began in September 2012 and finished in January 2014. All the objects were divided into bright $(\mathrm{B}, r=14.0 \sim 16.0 \mathrm{mag})$ and faint $(\mathrm{F}, r=16.0 \sim 18.1 \mathrm{mag})$ plates. The typical exposure times for $\mathrm{B}$ and $\mathrm{F}$ plates are $3 \times 600 \mathrm{~s}$ and $3 \times 1800 \mathrm{~s}$, and vary according to the weather condition and telescope performance. The galaxies with low $\mathrm{S} / \mathrm{N}$ ratio, quasars and $\mathrm{u}$-band variable sources are observed repeatedly, from twice to eleven times. We observed 2447 (out of 2519) galaxies in Field A and 2995 (out of of 3104) galaxies in Field B.

The raw data have been reduced with LAMOST 2D and 1D pipelines (Luo et al. 2004, 2012, 2014). To get reliable redshifts, an additional process is applied to 1D spectra. All the emission and absorption lines are checked and identified visually. The final uncertainties in redshifts are less than 0.001 .

\section{Preliminary Results}

In total, for 1528 of 2447 observed galaxies ( $62 \%)$ in Field A and 1573 of 2995 observed galaxies in Field B $(\sim 53 \%)$, we obtain reliable redshifts, ranging from 0.001 to 3.148. The distributions of redshifts (less than 0.3) of the two fields are shown in Figure 1.

Based on these data, we have obtained some preliminary results. First, we discovered 64 new LIRGs in LCSSPA Field A (Lam et al. 2015) and analyzed their infrared properties. Secondly, using the Gaussian Mixture Model, we determine the members of galaxy cluster Zwicky 721 and present its abnormal structure (Yang et al., in progress). Thirdly, we identified spectra of u-band variable objects at LCSSPA (Cao et al., in progress).

\section{References}

Abell, G. O., Corwin, H. G., Jr., \& Olowin, R. P. 1989, ApJS, 70, 1

Lam, M. I., Wu, H., Yang, M., et al. 2015, RAA, 15, 1424

Luo, A.-L., Zhang, Y.-X., \& Zhao, Y.-H. 2004, SPIE, 5496, 756

Luo, A.-L., Zhang, H.-T., \& Zhao, Y.-H., et al. 2012, RAA, 12, 1243

Luo, A., Zhang, J., Chen, J., et al. 2014, IAUS, 298, 428

Wu, X.-B. \& Jia, Z. 2010, MNRAS, 406, 1583

Wu, X.-B., Hao, G., Jia, Z., Zhang, Y., \& Peng, N. 2012, AJ, 144, 49

Zou, H., Wu, X. B., \& Zhou, X. et al. 2015, PASP, 127, 94 